\title{
The String Tension in Gauge Theories: a Suggestion for a New Measurement Method
}

\author{
Enzo MARINARI ${ }^{(1,2)}$, Maria Luigia PACIELLO ${ }^{(3)}$, \\ Giorgio PARISI ${ }^{(1,4)}$ and Bruno TAGLIENTI ${ }^{(3)}$ \\ (1): Dipartimento di Fisica and Infn, \\ Università di Roma Tor Vergata, \\ Viale della Ricerca Scientifica, 00133 Roma (Italy) \\ (2): Physics Department and NPAC, \\ Syracuse University, Syracuse, NY 13244 (USA) \\ ${ }^{(3)}$ : Infn Sezione di Roma \\ Università di Roma La Sapienza, \\ Piazzale Aldo Moro, 2 - 00185 Roma (Italy) \\ marinari, paciello, parisi, taglienti @roma1.infn.it
}

November 19, 2018

(4): Address after November 1st: Dipartimento di Fisica and Infn - Università di Roma La Sapienza, Piazzale Aldo Moro, 2 - 00185 Roma (Italy)

ROMA $92-903$

hep-lat/9210021 


\begin{abstract}
We discuss a new method for testing confinement and measuring the string tension (in the Coulomb gauge). Our numerical simulations demonstrate that the problems related to Gribov copies are not harmful and that the method is effective in the case of pure gauge Q.C.D.. We discuss the relevance of the method for studying gauge theories coupled to fermionic matter.
\end{abstract}


In this note we introduce a new method (following an en passant remark of ref. [四) for measuring the string tension $\sigma^{2}$ in gauge theories, and to establish a criterion for confinement. We show numerically the validity of this approach, and we discuss its relevance toward the simulation of fully coupled Q.C.D..

Our approach will be based on the use of Coulomb gauge; we will deal with the gauge fixed lattice theory, in a gauge that is smooth at fixed euclidean time, i.e. where the spatial gauge fields are brought, by gauge transformations, as close as possible to the identity.

We will consider in the following an $S U(3)$ gauge theory defined on a lattice of volume $L^{3}$ and time extent $T$, with periodic boundary conditions in the 4 dimensions.

The gaugeon of length $n$ is defined by

$$
G_{n}\left(\vec{x}, t_{0}\right) \equiv \prod_{t=t_{0}}^{t_{0}+n}\left[U_{t}(\vec{x}, t)\right]
$$

and we integrate over a spatial 2-plane (going to zero 2-momentum) by setting

$$
G_{n}\left(x_{\alpha}, t_{0}\right) \equiv \frac{1}{L^{2}} \sum_{\vec{x}_{\beta}, \vec{x}_{\gamma}} G_{n}\left(\vec{x}, t_{0}\right),
$$

where $\alpha, \beta, \gamma=1,2,3, \alpha \neq \beta \neq \gamma$. We compute the zero 2-momentum correlation functions by defining

$$
C_{n}(d) \equiv \frac{1}{3 L T} \sum_{\delta=1,2,3} \sum_{t_{0}=1, T} \sum_{x_{\alpha}=1, L}\left\langle G_{n}\left(x_{\delta}, t_{0}\right) G_{n}^{\dagger}\left(x_{\delta}+d, t_{0}\right)\right\rangle .
$$

Let us introduce the point by elaborating, in the form discussed in ref. [1] , a point originally developed by Ferrari and Picasso 24. The argument hints the relevance of measuring, in the Coulomb gauge, correlation functions of time-like gauge fields at the same time (and different spatial points), of the type (3).

The point of view suggests that the photon can be seen as the Goldstone boson of the gauge symmetry, and that the instantaneous potential $\frac{1}{x}$ (the Coulomb potential in Coulomb gauge) can bee seen as originated from the exchange of a Goldstone boson. For understanding the point let us consider 
our lattice theory, which has an invariance $S U(3)^{L^{3} T}$. This is the gauge symmetry of the lattice theory, and the gauge group is somehow too large to be broken from Goldstone bosons.

We can anyhow gauge fix our theory, and reduce the symmetry. Let us fix Coulomb gauge, by maximizing the expression

$$
\sum_{i} \sum_{\mu=1}^{3} \operatorname{Re}\left\{\operatorname{Tr}\left[U_{\mu}(x)\right]\right\} .
$$

We can get rid of Gribov copies in many different ways. We can define the expectation values by averaging over all copies with equal probability, or we can choose, with lot of work, the global maximum (which is generically unique). We could also assign to each copy a different weight which is proportional to its basin of attraction in the algorithm we are using to fix the gauge. We note that for similar gauge fixing algorithms the basins of attraction are quite similar.

Independently from the method we use to deal with Gribov copies, the crucial point is that now there is a residual symmetry. Gauge transformations which only depend on time but are space independent leave the quantity (4) invariant. This symmetry is a global $S U(3)$ for each time slice, i.e. the total residual symmetry is $S U(3)^{T}$. But on a fixed time slice (a sensible entity to consider in Coulomb gauge) now we have a global symmetry, which in the $V \rightarrow \infty$ can by broken generating a Goldstone boson. In the $V \rightarrow \infty$ limit the symmetry will be indeed broken in the Coulomb phase (where we will have a Goldstone boson for each time slice, and the expected propagator), while it will be preserved in the confined phase. $C_{1}(d)$ will tend to a constant for $d \rightarrow \infty$ in the Coulomb case, while it will decay exponentially in the confined phase.

This physical picture leads us to suggest to use $C_{n}(d)$ in order to measure the string tension. We expect that for $n$ and $d$ large enough $C_{n}(d)$ will decay, in the confined phase, with a behaviour

$$
e^{-\sigma^{2} n d}
$$

There are two ways which are usually employed to measure the string tension $\sigma^{2}$ and to distinguish between the confined and the deconfined phase. One is based on the measurement of large Wilson loops (the original Creutz ratios), while the other is based on measuring correlation functions of Polyakov 
loops. Statistical improvements, like for example the use of smeared looppy observables, turn out to be crucial (related to the fact we are working in a critical limit, where a correlation length is diverging).

In a pure gauge theory the expectation value of a Wilson loop of area $A=B \times H$ behaves as $e^{-A}$ if the theory is confined, and as $e^{-L}$ if the theory is deconfined. If we couple the theory to fermions we can close a fermion loop only paying a price proportional to the loop length, and we get again an $e^{-L}$ decay. So the Wilson loop ceases to be a good indicator when we deal with the full theory.

The Polyakov loop correlation function at distance $d$ behaves for large $d$ as $e^{-\sigma^{2} L d}$ in a confined pure gauge theory, and gets a non-zero connected part when the pure theory deconfines. Also in this case the fully coupled theory does not acknowledge a difference between the two phases, since also in the confined phase the fermion loops give a non zero expectation value to the loop-loop correlation function. The two most popular ways used to determine the string tension $\sigma^{2}$ and to distinguish between the two phases are not effective in the case of the theory coupled to fermions.

On the contrary we expect the gaugeon-gaugeon correlation functions $C_{n}(d)$ behave asymptotically (for large $d$ ) as $e^{-f(n) d}$ both in the pure gauge theory and in the theory coupled to fermions in the confined phase. In the case of the pure gauge theory we expect $f(n)$ to coincide (for large $n$ ) with $\sigma^{2} n$. Here indeed the $U$ cannot take an expectation value if the symmetry is unbroken. The method can be used both in the pure gauge and in the fermionic theory, and is likely to be a very effective method in both cases. In the following we will discuss a pure gauge numerical simulation in which we demonstrate its effectiveness.

We have analyzed 100 configurations on a $10^{3} \times 20$ lattice and 412 configurations on a $10^{3} \times 6$ lattice, both at $\beta=5.8$. They have been separated (after 2000 thermalization sweeps) of 500 sweeps of an 8 hit Metropolis updating scheme. Coulomb gauge has been fixed by using an over-relaxed procedure. On each independent gauge configuration we have gauge fixed ten times, starting from 10 different randomly gauge transformed samples. We were interested to check if Gribov copies can have an influence of such a quantity (since it is computed in a gauge fixed environment). So we have averaged separately the configurations which turned out to have a maximum value of (田), the medium ones and the minimum ones. We have independently computed the rate of the 3 decays, and in the limit of our statistical error we 
have not seen any difference between them.

In fig. 1 we show the effective mass estimator, as defined from the logarithm of the ratio of two $C_{n}(d)$ (with the corrections needed from the presence of periodic boundary conditions) for our largest lattice. We have points for the ration of distance 1 and 2,2 and 3 and 3 and 4 . The lines give our best fit, of the form

$$
\sigma_{(n)}^{2}=\sigma_{(\infty)}^{2}+\frac{c_{1}}{n}
$$

which turns out to be perfect in all cases. Here measuring directly an estimator for the string tension (also at distance 1 and 2, which is however highly biased, since we are used local, non-smeared Wilson loops) is impossible, since the lattice is too large (the time asymptotic result is of order 0.1 , see for example [3]). In order to stress the very good linearity of our data as a function of the gaugeon size $n$ we plot the effective mass as a function of $n$ in Fig. 2.

In Figs. 3 and 4 we plot the same data for the $10^{3}$ lattice. Here we can compute the true estimator for $\sigma_{\text {effective }}^{2}$ at distance 1 over 2 , and we find that there is a small systematic difference from the curve extrapolated by using the non-gauge invariant gaugeons. We expect such a small systematic effect, which will tend to zero in the continuum limit, since this is a gauge invariant measurement. This additional systematic error has to be kept under control, but does not seem to be a dangerous effect, already at a quite low value of $\beta$.

This result is very satisfactory, as far as the $n$-dependence of the effective mass estimator at fixed distance seems to be under very good control. In the limit of our statistical precision The presence of Gribov copies seems to be irrelevant as far as our results do not depend on criterion we have used to choose the copies. Obviously in a practical implementation of the method one will limit is search to a single gauge fixed copy for each independent starting gauge configuration (where, at $\beta=5.8$, the decorrelation time for local observables will be surely smaller than the one we used here to be sure to get rid of all non-local correlations).

As far as we have been able (by using non-smeared Wilson loops) to measure the effective mass only up to distance 3 over 4 the extrapolation of the effective mass estimator at large distance is problematic. We can only notice that the estimator from times 3 over 4 (extrapolated at large $n$ ) is 
about the $50 \%$ higher than the asymptotic value of the string tension: this is the best upper bound to the value of $\sigma^{2}$ we have been able to obtain and is a quite reasonable result. We expect the use of smeared operators to be very effective in decreasing the error. Checking how effective the method is when dealing with smeared operators seems to be the next important step. 


\section{References}

[1] G. Parisi, A Short Introduction to Numerical Simulations of Lattice Gauge Theories, in Phénomènes Critiques, Systèmes Aléatories, Theories de Jauge, p. 87, edited by K. Osterwalder and R. Stora (NorthHolland, Amsterdam, The Netherlands 1986).

[2] R. Ferrari and R. Picasso, Nucl. Phys. B31 (1971) 316.

[3] E. Marinari, Nucl. Phys. B (Proc. Suppl.) 9 (1989) 209. 


\section{Figure Captions}

Fig. 1 Distance dependent effective mass estimator as a function of the gaugeon size $n .10^{3} \times 20$ lattice. Continuous lines are the best fits.

Fig. 2 As in fig. 1, but as a function of $n^{-1}$.

Fig. 3 As in fig. 1 , but $10^{3} \times 6$ lattice.

Fig. 4 As in fig. 1 , but $10^{3} \times 6$ lattice and as a function of $n^{-1}$. 